Review

\title{
Understanding the pathogenesis of spondyloarthritis
}

\author{
Aigul Sharip ${ }^{1}$ and Jeannette Kunz ${ }^{1, *}$ \\ 1 Department of Biology, School of Sciences and Humanities, Nazarbayev University, Nur-Sultan, Kazakhstan; \\ email. A.sharip@nu.edu.kz \\ * Correspondence: jeannette.kunz@nu.edu.kz; Tel.: +7-702-411-0463
}

\begin{abstract}
:
Spondyloarthritis comprises of a group of inflammatory diseases of the joints and spine with various clinical manifestations. The group includes ankylosing spondylitis, reactive arthritis, psoriatic arthritis, arthritis associated with inflammatory bowel disease, and undifferentiated spondyloarthritis. The exact etiology and pathogenesis of spondyloarthritis are still unknown, but five hypotheses explaining the pathogenesis exist. These hypotheses suggest that spondyloarthritis is caused by arthritogenic peptides, an unfolded protein response, HLA-B27 homodimer formation, malfunctioning endoplasmic reticulum aminopeptidases, and, last but not least, gut inflammation and dysbiosis. Here we discuss the five hypotheses and the evidence supporting each. In all of these hypotheses, HLA-B27 plays a central role. It is likely that a combination of these hypotheses, with HLA-B27 taking center stage, will eventually explain the development of spondyloarthritis in predisposed individuals.
\end{abstract}

Keywords: spondyloarthritis, HLA-B27, pathogenesis, inflammation, arthritogenic peptides, unfolded protein response, ERAP1, gut dysbiosis 


\section{Introduction}

Spondyloarthritis (SpA, also called spondyloarthropathy) is a group of inflammatory diseases of the joints and spine with various clinical manifestations. Five major subtypes of SpA are recognized on the basis of the classification criteria proposed by the European Spondyloarthropathy Study Group (ESSG) [1]. These subtypes include ankylosing spondylitis (AS), reactive arthritis (ReA), psoriatic arthritis (PsA), arthritis associated with inflammatory bowel disease (SpA-IBD), and undifferentiated spondyloarthritis (uSpA) [2,3]. More recently, a classification into axial and peripheral diseases has been proposed. The main clinical manifestations of SpA are inflammatory pain and stiffness and swelling of thejoints and spine. In addition, peripheral arthritis, enthesitis, dactylitis, sacroiliitis, and chronic inflammatory bowel disease (IBD) are often observed [3,4]. Less common symptoms are inflammation of the eye or GI tract, and hearing loss [5].

The exact etiology and pathogenesis of spondyloarthritis are still unknown. However, several lines of evidence indicate that genetics plays an important role in individuals' susceptibility, while environmental factors -resulting in infections and gut dysbiosisalso contribute to SpA pathogenesis. Various studies demonstrated that microorganisms play a role in triggering the disease. Genetically, SpA is strongly associated with the major histocompatibility complex (HMC) class I antigen, HLAB27. For example, more than $95 \%$ of patients with AS possess HLA-B27, whereas its frequency among the general population is less than $10 \%$. The risk of developing AS is as high as 5-7\% in HLA-B27-positive individuals [5]. HLA-B27 consists of an alpha chain encoded in the B locus of the MHC region on chromosome 6 and a non-MHC encoded beta chain, $\beta_{2}$ microglobulin (Figure 1). The primary function of HLA-B27 in complex with $\beta 2$-microglobulin is to present short antigenic peptides for recognition to cytotoxic T lymphocytes (CTLs; Figure 1). The ability of HLA-B27 to bind a unique set of peptides may be the underlying reason for its disease association with SpA [6,7]. Three unique features of HLA-B27, including peptide binding specificity, a tendency 
to misfold, and the predisposition for forming heavy chain homodimers may contribute to disease pathogenesis [8]. Each of these features has been suggested independently to play a role in the pathogenesis of SpA.

Moreover, HLA B-27 is a highly polymorphic molecule of which currently (April 2020), more than 223 subtypes are known (https://www.ebi.ac.uk/ipd/imgt/hla/allele.html). The high number of polymorphisms, in combination with heterozygosity, give a selective advantage to the immune system against the diversity of microorganisms and their antigens. However, extreme polymorphism and potential mutations in the major histocompatibility complex (MHC) increase the chance of developing autoimmune diseases [9]. Thus, these molecules play an essential role in the regulation of the host's immune response by initiating tolerance and CTL or helper $\mathrm{T}$ cell responses by presenting peptides to T-cell receptors [10,11]. Among the HLA-B27 alleles, HLA$B^{*} 2705$ and HLA-B*2704 are the most strongly associated with SpA, whereas, HLA$B^{*} 2706$ and $B^{*} 2709$ are weakly associated [12]. It is well known that the prevalence of SpA varies strongly between ethnic groups and populations, this can partly be explained by differences in the prevalence of HLA-B27 $[3,13]$. The prevalence of AS varies from $0.2 \%$ to $0.7 \%$ among European populations while higher frequencies of AS have been reported in populations from Siberia, Alaska, and Scandinavia [13].

The interaction between bacteria and HLA-B27 alleles plays an important role in the hypotheses of SpA pathogenesis [14]. The evidence that ReA is triggered by Chlamydia trachomatis (genitourinary infections) or by other gram-negative enterobacteria, such as Shigella, Salmonella, Yersinia, and Campylobacter species provides a solid background for these hypotheses [15]. Also, inflammatory arthritis frequently develops following infection with enteric organisms such as Clostridium difficile, Brucella, and Giardia. The identification of microbial antigens in the synovium of $\operatorname{ReA}$ indicates that those antigens can be crucial for ongoing joint inflammation [16]. The fact that some SpA types can be triggered by bacterial infections proves the importance of these organisms in SpA pathogenesis. Therefore, inappropriate immune responses to commensal 
bacteria or alterations in gut microbiota were also proposed to be factors in SpA development.

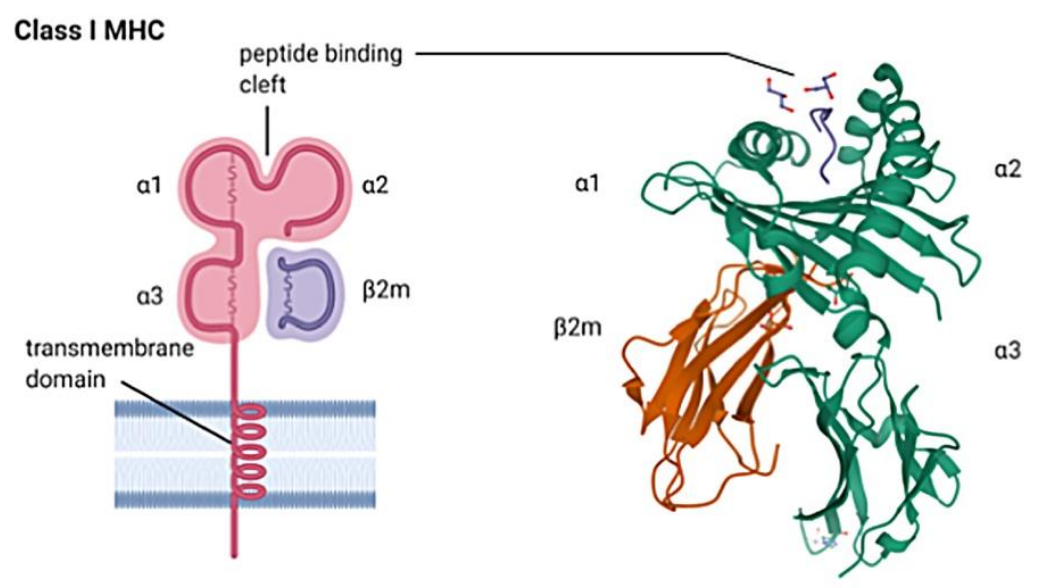

Figure 1. A model depicting the MHC Class I HLA-B27 heavy chain in complex with beta 2-macroglobulin ( $\beta 2 \mathrm{~m})$ The alpha $1(\alpha 1), 2(\alpha 2)$, and $3(\alpha 3)$ domains of the HLAB27 molecule are indicated on the left. The transmembrane domain inserted into the lipid bilayer of the plasma membrane and the peptide binding cleft composed of $\alpha 1$ and $\alpha 2$ domains of HLA-B27 are indicated by solid black lines. A ribbon model of the HLA-B27 heavy chain structure (green) with bound peptide and in complex with beta 2-macroglobulin ( $\beta 2 \mathrm{~m}$; orange) is shown on the right. The alpha $1(\alpha 1), 2(\alpha 2)$, and 3 (a3) domains of the HLA-B27 molecule are indicated. The peptide binding cleft composed of $\alpha 1$ and $\alpha 2$ domains of HLA-B27 is indicated. The bound peptide is shown. Orientation: cell surface at top of picture.

\section{Models of SpA pathogenesis: hypotheses}

The exact pathogenetic role of HLA-B27 is unknown [17]. There are, however, some predominant theories explaining the role of the HLA-B27 allele in SpA and disease pathogenesis, including the arthritogenic peptide hypothesis, misfolding protein hypothesis, as well as the hypothesis of the cell-surface HLA-B27 homodimer [18].

Numerous hypotheses have been suggested regarding the role of HLA-B27 in the pathogenesis of AS. These are mainly based on rodent animal models. One of the 
oldest and major theories for explaining HLA-B27 pathogenicity, is the so-called "arthritogenic peptide" hypothesis. This hypothesis proposes that the presentation of unique sets of antigenic peptides, either self- or bacteria-derived, by HLA-B27 alleles to CD8 T cells, results in cross-reactivity and an HLA-B27-restricted cytotoxic T-cell response. This response subsequently triggers a harmful CD8+ $\mathrm{T}$ cell-mediated response in joints and other affected tissues [13,19-22]. The second hypothesis, the "unfolded protein response," suggests that HLA-B27 tends to fold slowly and often misfolds. The misfolded HLA-B27 molecules accumulate in the endoplasmic reticulum (ER), where they induce a stress response that leads to an unfolded protein response (UPR) and autophagy [20-22]. The upregulation of UPR genes subsequently activates the production of pro-inflammatory cytokines such as IL-17, IL-23, and IFN- $\gamma$ [21]. Another hypothesis that explains the role of HLA-B27 in SpA pathogenesis is the "formation of homodimers" hypothesis. According to this hypothesis, HLA-B27 heavy chains homodimerize at the cell surface, where they act as pro-inflammatory ligands for humoral or cell-mediated autoimmune responses [12,20]. These homodimers are capable of binding to certain killer cell immunoglobulin-like receptors (KIRs), which are expressed on natural killer cells and T cells, and this leads to the upregulation of the pro-inflammatory cytokine IL-17 [22].

The gene with the second strongest SpA association, Endoplasmic Reticulum Aminopeptidase 1 (ERAP1), trims peptides to optimal sizes for antigen presentation. Loss-of-function alleles of ERAP1 lead to abnormal HLA-B27 presentation and accumulation of intracellular free heavy chains, which can result in changes in peptide processing with pathological consequences $[23,24]$. Non-genetic contributors to SpA are certain infections and gut dysbiosis. Many studies indicate a link between intestinal bacterial composition, in particular gut dysbiosis, and SpA. In this review, we will discuss the hypotheses to explain the disease mechanisms of SpA pathogenesis. We will also propose a hypothesis that combines the previously proposed hypotheses. 


\subsection{Arthritogenic peptide hypothesis}

The arthritogenic peptide hypothesis is one of the oldest hypotheses explaining the association between HLA-B27 and SpA [25]. It proposes that some HLA-B27 subtype alleles, due to their characteristic amino acid residues, bind a specific arthritogenic peptide that is recognized by CD8+ T cells [26]. This hypothesis assumes that the natural ligands of HLA-B27 are target antigens of autoimmune T cells induced by crossreactive bacterial peptides. Peptide presentation by HLA-B27 is affected by subtype polymorphisms that affect the peptide-binding groove [27]. Consequently, a set of unique antigenic peptide epitopes with key conserved anchor residues are recognized by specific HLA-B27 alleles. The arthritogenic peptide hypothesis is based on the peptide binding specificity of HLA-B27, the concept of molecular mimicry, and the idea that activation of $\mathrm{T}$ cell responses by an external antigen may result in autoimmunity. The initial pathogenic event in SpA could be the activation of HLA-B27 by CTL responses against bacterial peptides. Cross-reaction of some activated CTL with a self-peptide would subsequently lead to autoimmune tissue damage and inflammation [28].

Various studies support the arthritogenic peptide hypothesis. F or in st an ce, HLAB27-restricted CD8 T cell responses specific for Salmonella or Chlamydia have been identified in patients who developed reactive arthritis following infections with these pathogens [29,30]. Also, B27- restricted CD8+ CTL were found in the synovial fluid of ReA patients [31,32]. A set of peptides derived from enteric organisms were identified that bound to and have sequence homology with, HLA-B27 [33]. AS patients also have a higher probability of having autoantigenic antibodies directed against Klebsiella that are cross-reactive against B27 in comparison to HLA-B27 positive but healthy individuals [34]. Moreover, investigation of the HLA-B27 peptidome identified more than a thousand peptides from HLA-B27+ cell lines, half of which were found to tightly bind to HLA-B27 alleles. Twenty eight of these peptides were considered to be arthritogenic peptides as they were derived from cartilage or bone proteins that had 
high homology with proteins from enteric bacteria. These findings suggest that these antigenic peptides can induce a T-cell mediated immune response that results in SpA pathogenesis $[25,35]$. Along with bacterial peptides, viral peptides could also contribute to SpA pathogenesis. In some SpA patients, cross-reactive CD8 T cell responses that recognized both an epitope from Epstein-Barr virus (EBV) and a self-peptide derived from the vasoactive intestinal protein receptor (VIPR) were identified [36]. However, EBV infection is not recognized as a SpA trigger and these findings warrant further investigation. Major support for the arthritogenic peptide hypothesis comes from studies of HLA-B27 alleles and their differential association with SpA pathogenesis. For example, some alleles such as HLA-B27*05, HLA-B27*02, and HLA-B27*04 are strongly associated with the disease, whereas some other alleles (HLA-B27*06 and HLA-B27*09) were not or only weakly associated with disease pathogenesis [37].

Nevertheless, conclusive evidence that directly implicates this mechanism in the pathogenesis to support this arthritogenic peptide hypothesis is still lacking [20]. Recently, HLA-B27 peptidomes from SpA-related and unrelated alleles were studied, and no clear distinction in qualitative differences between these groups was found, indicating that binding preferences of HLA-B27 alleles do not solely explain disease association $[38,39]$.

\subsection{The unfolded protein response hypothesis}

The unfolded protein response (UPR) hypothesis is another theory explaining the role of HLA-B27 in SpA pathogenesis. Compared with other MHC molecules, HLA-B27 tends to slowly fold or even misfold in the endoplasmic reticulum (ER), and to accumulate in the ER for extended periods of time, which may have implications for pathogenesis [40]. The accumulation of misfolded forms of HLA-B27 in the ER leads to ER stress that induces the UPR, which is a homeostatic mechanism that aims to return the cell to its normal state [41]. 
UPR suppresses protein translation and upregulates ER chaperone molecules such as immunoglobulin heavy-chain-binding protein $(\mathrm{BiP})$ and endoplasmic reticulumlocalized DnaJ 4 (ERdj4). It also activates transcription factors such as CCAATenhancer-binding protein homologous protein (CHOP) and increases the production of pro-inflammatory cytokines (IL-23, INF- $\beta$, and IL-1) [42]. Indeed, UPR affects cytokine production on multiple levels, from stimulation of surface receptors to activation of inflammatory signaling pathways, in particular the IL-23/IL-17 signaling pathway [43]. Autophagy induced by ER stress and UPR also increase the production of IL-23 [44].

Misfolded HLA-B27 molecules in the ER are disposed of by ER-associated degradation (ERAD), therefore, slow folding or accumulation of misfolded HLA-B27 increases levels of ERAD and stimulates UPR and autophagy, in particular during inflammation when HLA-B27 production is high [44,45].

The slow-folding kinetics and partial misfolding of HLA-B*2705 lead to the activation of NF-kB, which leads to increased production of pro-inflammatory cytokines, such as TNF, IL-1, and IL-6. This hypothesis is concordant with observations in transgenic mice showing that the absence of $\beta 2$ microglobulin is required for the development of arthritis [46,47].

In support of the UPR hypothesis, it was shown that misfolding and UPR occur in HLA-B27 transgenic rat models of AS and in the gut and synovial tissues of SpA patients [48]. Bone marrow-derived macrophages from HLA-B27 transgenic rats show evidence of HLA-B27 misfolding after cytokine stimulation and this was associated with IL-23 production [49,50]. These HLA-B27 transgenic rats have functional alterations in the number of cell populations, which in turn can be correlating with misfolding. Moreover, the introduction of additional copies of the human $\beta 2-$ microglobulin gene reduced B27 misfolding increases the incidence and severity of arthritis [51]. Nevertheless, studies of tissues of AS patients did not suggest that UPR and ER stress were associated with inflammation and HLA-B27 misfolding [52,53]. 
Therefore, HLA-B27 was found to lead to misfolding and ER stress in both in vitro and animal studies; however, currently, there is no evidence of involvement of this process in SpA pathogenesis in humans.

It was demonstrated that in M-CSF-derived macrophages from AS patients, UPR pathway genes (CHOP and $\mathrm{BiP})$ and IL-23 are upregulated in comparison to healthy controls [54]. Recently, it was suggested that autophagy but not UPR is responsible for the regulation of IL-23 expression in the gut of AS patients [53]. This hypothesis is further supported by the observation that disease severity is unaffected by the lack of functional CD8+ T cells but requires CD4+ T cells in HLA-B27 transgenic rats [37,55].

HLA-B27 misfolding and UPR cellular environments are associated with enhanced Salmonella replication. It was demonstrated that the misfolding of HLA-B27 occurred without significant UPR activation in the gut of AS patients [56]; nevertheless, elevated BiP expression and UPR activation were observed in synovial fluid and cytokineinduced macrophages of AS patients $[57,58]$.

\subsection{HLA-B27 homodimer formation hypothesis}

The ability of HLA-B27 to form disulfide-bonded homodimers is one of the unique characteristics of this molecule. These homodimers are expressed on the cell surface of a variety of cell types, including natural killer cells (NKs), CTLs, and B lymphocytes [12]. It has been suggested that homodimer formation is a sign of HLA-B27 misfolding within the ER and that the accumulation of misfolded protein potentially results in a pro-inflammatory stress response [40]. HLA-B27 homodimers can facilitate the production of Th17 cells and can also upregulate the expression of IL-23/IL-1 in the Th17 cells [41]. HLA-B27 homodimers can bind KIRs and leucocyte immunoglobulinlike receptors (LILRs), which are expressed on NKs and T cells, and thus upregulate the IL-23/IL-17 signaling pathway and inflammation [22,59].

The ER protein misfolding has a variety of biological effects depending on the nature 
and quantity of the misfolded proteins, as well as on the severity of the defect. UPR activation can alter cytokine production, including increased production of proinflammatory cytokines IL-27, which is essential for SpA pathogenesis. Altered production of TNF and IFN- $\gamma$ may play a crucial downstream role that contributes to the unique spondyloarthritis phenotype [8].

There is much evidence supporting the HLA-B27 homodimer hypothesis. Both KIRs and LILRs play crucial roles in immune responses, including differentiation of macrophages and dendritic cells, T-cell survival, and activation of regulatory $\mathrm{T}$ cells (Tregs) [60]. The KIR3DL2 receptor was shown to recognize HLA-B27 homodimers with higher affinity than normal HLA-B27 heterodimers [18]. So far, seventeen KIR genes have been identified, and interaction of KIR3DL2 with HLA-B27 free heavy chains was shown to have pro-inflammatory effects in NK and T cells, and was linked to elevated Th17 expression in AS patients [59,61,62]. The binding of KIR3DL2 with HLA-B27 homodimers was also observed to facilitate the survival and differentiation of CD4+ $\mathrm{T}$ cells in SpA patients. In addition, it increased production of proinflammatory cytokines such as IL-17, TNF, and IFN- $\gamma[18,60,63]$. Moreover, in HLAB27 positive SpA patients, KIR3DL21 NK cells and CD41 T cells were increased in peripheral blood and showed a higher level of cytotoxicity and IL-17 production than in HLA-B27 negative SpA patients [57,64]. Thus, the binding of HLA-B27 homodimers to KIR and LILR facilitates inflammation by enhancing the survival of NK and T cells and affecting the differentiation of LILR-expressing antigen-presenting cells $[13,37,60]$. In addition, the arthritis-associated HLA-B²7:05 allele was shown to form more cellsurface homodimers than the non-associated HLA-B*27:09 allele [65].

Nevertheless, some research suggests that HLA-B27 homodimers are not very important in SpA pathogenesis. The ability to form homodimers of eight common HLA-B27 alleles (HLA-B*27:02 to HLA-B*27:09) was analysed and no major differences were found between disease-associated and non-associated HLA-B27 allotypes. In particular, HLA-B*27:03, an arthritis-associated HLA-B27 subtype, has a 
reduced ability to form cell-surface homodimers. These findings challenge the role of HLA-B27 homodimer formation in SpA pathogenesis and suggest that other features of HLA-B27 play a more central role in disease mechanism [66].

\subsection{The hypothesis of malfunctioning ERAPS}

The genetic association of polymorphisms in the ER aminopeptidases, ERAP1 and ERAP2, with AS is the second strongest after HLA-B27 and contributes approximately $15-25 \%$ of the population risk [23,67]. This association was first reported in the UK and the USA and was validated in independent studies with other populations [21,68-70]. Together, HLA-B27 and ERAP explain 70\% of the genetic risk of developing SpA [71,72]. Endogenous proteins presented to MHC I class molecule are transported to the ER and trimmed to a suitable length for MHC-I binding by ERAP1 and ERAP2 [73]. ERAP-1 is a non-MHC SpA associated gene and member of the zinc metallopeptidases, which is involved in antigen cross-presentation and regulates the shedding of tumor necrosis factor receptor 1 (TNFR1) [74].

ERAP1 has two major functions. The first is trimming of processed N-termini of peptides in the ER to a suitable length (8-10-mer) to allow binding to MHC class I molecules and presenting them on the cell surface of T cells. A failure in this function of ERAP1 causes changes in the normal expression of HLA-B27 and increases intracellular free heavy chain forms of HLA-B27 in antigen-presenting cells $[39,75]$. The second function of ERAP1 is the cleavage of cytokine receptors that are expressed on the cell surface, such as TNFR1, IL6R2, and IL1R2, which leads to a reduction of proinflammatory signaling [68,76]. These cytokine receptors play, however, no major role in SpA development [69,77].

The fact that the strong genetic association of AS with ERAP1 was only found in HLAB27 positive individuals indicates that ERAP1 most likely interacts with HLA-B27 within the antigen-processing and presentation pathway $[36,67]$. So, abnormal peptide 
processing and antigen presentation is a crucial contributor to SpA pathogenesis [69,78]. Epistatic interactions with ERAP1 were also identified for Behçet`s disease with HLAB51 [79], and for psoriasis with HLA-Cw3 [80]. These interactions suggest the possibility of similar HLA-driven pathogenic mechanisms in these diseases. Moreover, it was shown that ERAP1 polymorphisms can also affect the accumulation of HLA-B27 homodimers on the cell surface [37,61].

The exact mechanism behind the genetic association with ERAP1 is unclear. Nevertheless, it was found that various ERAP1 polymorphisms contribute to the altered disease risk. For example, rs30187, a polymorphism that changes the amino arginine to the more enzymatically active lysine at position 528 (R528L), increases disease risk [81]. In addition, loss-of-function ERAP1 polymorphisms affect HLA-B27 heavy chain dimerization and misfolding [69,81]. Moreover, AS patients appear to have higher expression of the ERAP1 gene (Campbell et.al 2011), so overexpression of ERAP1 may be one of the mechanisms explaining SpA pathogenesis. More importantly, it was demonstrated that the loss of ERAP1 function in humans and mouse models leads to changes in the MHC I peptidome, indicating it is important for shaping the MHC I peptide repertoire [82]

ERAP1 polymorphisms may play a key role in SpA pathogenesis through variation in the peptide repertoire bound to HLA-B27, and through the generation of abnormal intracellular and extracellular forms of HLA-B27 by affecting either ER misfolding or the export of pro-inflammatory B27 forms [83]. Various ERAP1 polymorphisms have been identified that are associated with the disease [84]. For example, the ERAP polymorphisms rs30187, rs27044, and rs2287987 were associated with AS susceptibility in a Polish population [85]. In an Iranian population, the ERAP1 polymorphisms rs30187 and rs2287987 were associated with the risk of developing AS [24]. Interestingly, the polymorphism rs75862629 provided protection from AS in HLAB27 positive individuals in Sardinia by regulating the expression of both ERAP1 and ERAP2 [86]. However, the effect of ERAP1 polymorphisms on the susceptibility to SpA 
may vary among ethnic groups; therefore, identification of genetic variations in different populations could be important for understanding SpA pathogenesis [24,84].

The association between ERAP1 polymorphisms and the development of AS also supports the arthritogenic peptide hypothesis. The function of ERAAP (the ERAP1 equivalent in mice) is, in mice, important for MHC class I peptide processing and presentation, and its destruction leads to an abnormal CD8 $\mathrm{T}$ cell response [87]. Moreover, ERAP1 polymorphisms might be associated with SpA by increasing TNF receptor shedding [76], there were, however, significant differences in mRNA expression of certain cytokines between AS patients with different ERAP1 haplotypes $[24,88]$.

In addition to ERAP1 polymorphisms, ERAP2 polymorphisms may also be associated with AS, despite that ERAP2 is not involved in receptor shedding [25]. In addition, ERAP1 and ERAP2 polymorphisms are associated with a variety of inflammatory diseases, including AS. The relationship between ERAP1 and HLA-B27 might be crucial for SpA development, as it was shown that the inhibition of ERAP1 leads to changes in cells with disease-associated HLA-B*27:04 or HLA-B²7:05, but not in cells with the non-associated HLA-B*27:06 or HLA-B*27:09 [21].

In summary, some longer peptides that are generated by abnormal peptide processing due to defective ERAP1 play a crucial role in the pathogenesis of SpA by changing the antigen presentation pathway, resulting in activation of an inflammatory and pathogenic immune response [24,89].

\subsection{Gut inflammation and dysbiosis hypothesis}

The most recent hypothesis explaining SpA pathogenesis is the role of HLA-B27 in shaping the gut microbiome, and HLA-B27-mediated changes in the gut microbiome associated with disease susceptibility [39]. The gut microbiome is a complex homeostatic ecosystem consisting of trillions of bacteria, and plays important roles in 
the development of the host's immune system, in food digestion, and the intestinal epithelial barrier $[39,90]$. Changes in gut microbiome composition are correlated with autoimmune diseases through mechanisms such as the activation of the immune response, molecular mimicry, and increased intestinal permeability [39].

HLA-B27 could influence SpA pathogenesis by shaping the gut microbiome. There are multiple lines of evidence supporting this theory. It was found that HLA-B27 facilitated the survival of Gram-negative intracellular bacteria in HLA-B27 expressing HeLa cell lines [91,92]. There is a known correlation between host genetic background and changes in the gut microbiome that establish an inflammatory state which can promote arthritis [44,93]. The gut microbiome in HLA-B27 transgenic rats is significantly different from the gut microbiome in littermate controls [21,94] The relationship between the gut microbiome and arthritis has been actively investigated in human studies [44].

Moreover, it was hypothesized that HLA-B27-induced changes in the gut microbiome can be an important step in the development of SpA [90,95]. It was shown that gut dysbiosis and inflammation could facilitate innate immunity activation and Th17 cell expansion in HLA-B27 transgenic rats [96]. A lot of evidence supports the relationship between gut inflammation and SpA. For example, $7 \%$ of AS patients also have IBD. Conversely, $10-50 \%$ of IBD patients develop SpA [90]. Therefore, it is crucial to understand how HLA-B27 shapes the gut microbiome and impacts SpA development.

The advent of genomic technologies and advancement in bioinformatic analyses will facilitate the comprehensive understanding of gut microbiome changes of SpA patients and have already facilitated significant improvements in this field [39]. Various studies have shown that the presence of specific changes in the gut microbiome can be responsible for the initiation of disease, such as an increased number of opportunistic pathogens, gut dysbiosis, and a shift in the composition of commensal bacteria [97,98]. Gut dysbiosis could trigger antigenic activation of pathogenic effector cells of the immune system, which in turn facilitate chronic 
inflammation. Increased gut permeability is a characteristic and pathogenic pathway of SpA development. Tight junctions between intestinal epithelial cells are dysregulated, which leads to increased gut permeability and harms mucosal immunity by altering normal regulation of gut microbiota and secretion of pro-inflammatory cytokines [99]. The evidence for this hypothesis is that intestinal permeability is increased in AS patients and that bacterial by-products have been detected in the synovial fluid of joints of AS patients [100].

Through various metagenomics studies, the gut microbiome composition of SpA patients was demonstrated to be different from healthy controls. For example, a higher number of Lachnospiraceae, Veillonellaceae, Prevotellaceae, Porphyromonadaceae, and Bacteroidaceae was observed in patients with AS [101,102]. A metagenomics study analysed the gut microbiome composition in three cohorts of volunteers: SpA patients, RA patients, and healthy controls. A disease-specific dysbiosis was identified in SpA and RA patients, but not in healthy controls; in particular, a significantly increased amount of Ruminococcus gnavus were found in SpA patients, and its levels were correlated with IBD severity in this patient cohort. Moreover, significantly different gut microbiome compositions were detected in healthy HLA-B27 positive and negative individuals, which suggests that genetic background might affect gut microbiota [103]. Deep shotgun sequencing of 211 Chinese individuals identified 23,709 genes and 12 metagenomic species that were differentially expressed between AS patients and healthy controls. Prevotella melaninogenica, Prevotella copri and Prevotella sp. were increased while Bacteroides spp. were decreased in AS patients $[98,104]$.

There is substantial evidence suggesting that the interaction between host genetics and the gut microbiome is a key driver of the pathogenesis of SpA [105]. Pathogenic bacteria and possibly viruses occurring in the gut of SpA patients contribute to dysbiosis and lead to immune dysfunction and modulation of the innate and adaptive immune responses [98]. 


\section{Conclusions}

Despite intensive research, the exact mechanism of SpA pathogenesis and the pathogenic role of HLA-B27 remain unclear. Five major hypotheses each explain the role of HLA-B27 in SpA: the arthritogenic peptide hypothesis, the misfolded HLA-B27 hypothesis, the cell-surface homodimer formation hypothesis, the hypothesis on malfunctioning of ERAPs, and the hypothesis based on gut microbiome changes. In the previous sections, these hypotheses were described, and it was shown that there is ample evidence to support each of these. It is possible that all or most of these mechanisms contribute to disease in individuals. Moreover, taking into account that the frequencies of HLA-B27 alleles and ERAP1 polymorphisms are ethnic-specific, it is important to understand that SpA pathogenesis could well be the result of different combinations of these mechanisms in different populations (Figure 2).

The link between these hypotheses could be canonical and non-canonical features of HLA-B27 and ERAP1 polymorphisms. In particular, ERAP1 polymorphisms may contribute to all three models (arthritogenic peptide theory, unfolded protein response, and homodimer formation) that explain how HLA-B27 causes AS. ERAP1 could contribute to SpA by altering antigen presentation due to abnormal aminopeptidase activity and dysregulation of peptide processing. Altered rates of peptide trimming mediated by ERAP1 could lead to cell surface presentation of aberrant peptides for the HLA-B27 ligand. This facilitates modulation of the immune response through pro-inflammatory cytokines. Recently, ERAP1 variants have been reported to alter levels of HLA-B27 free heavy chains on the cell surface. Altered enzyme activity could thus affect the rate at which MHC-peptide complexes fold within the ER and contribute to the UPR and ER stress. Malfunctioning of ERAP1 could facilitate the accumulation of abnormally folded HLA-B27 in the ER, leading to the activation of the unfolded protein response and a downstream immune response, such as through the upregulation of IL-23. 
In conclusion, it is highly likely that more than one of these hypotheses is involved in SpA pathogenesis. This is strengthened by the fact that in each of these hypotheses HLA-B27 plays a central role.

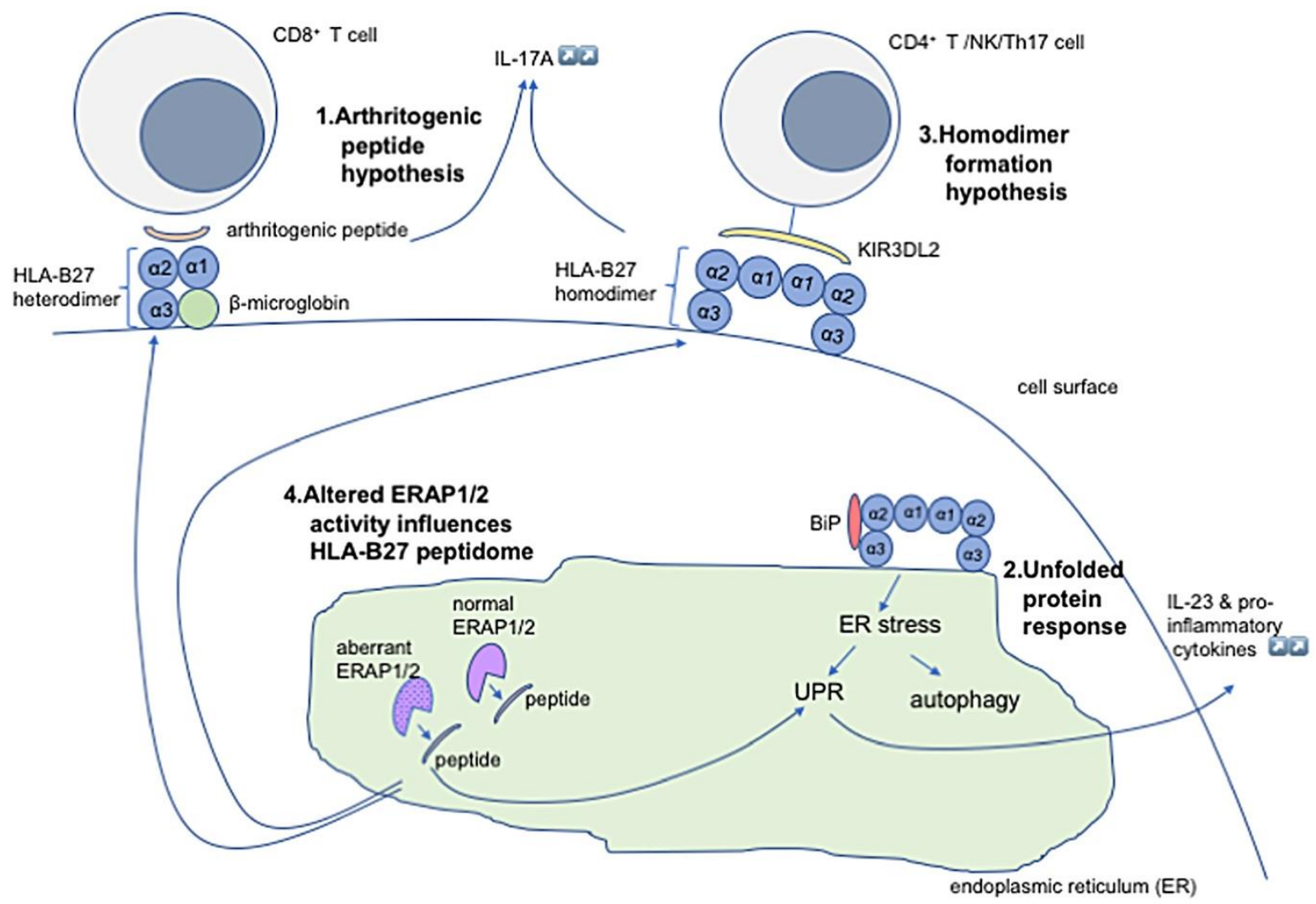

Figure 2. Illustration of the hypothesis for the pathogenetic role of HLA-B*2705 molecules in spondyloarthritis. (1) Arthritogenic peptides displayed by properly folded HLA-B27 can be recognized by autoreactive CD8+ T cells, resulting in inflammation (2) Misfolded HLA-B27 chains and binding of BiP causes ER stress and activation of UPR, leading to increased production of IL-23 and other proinflammatory cytokines (3) Cell surface HLA homodimers interreact with CD4+ T cells through innate immune receptors such as KIR3DL2 and facilitate cell-mediated autoimmune responses (4) Altered ERAP1 activity can result in changes in peptide processing with pathological consequences. ER, endoplasmic reticulum; ERAP1, ER aminopeptidases (ERAP)1; KIR3DL2, killer immunoglobulin-like receptor; UPR, unfolded protein response. 


\section{Author contribution}

A.-S.; conceptualization, writing-original draft preparation, J. K.; writing - review and editing; program supervision; funding acquisition.

\section{Funding}

This work was funded by a Nazarbayev University research award (Project number 064.01.01 SST 2016022 to JK) and by personal funds.

\section{Conflict of interest}

The authors declare no conflict of interest.

\section{References}

1. Zochling, J.; Brandt, J.; Braun, J. The current concept of spondyloarthritis with special emphasis on undifferentiated spondyloarthritis. Rheumatology (Oxford) 2005, 44, 1483-1491, doi:10.1093/rheumatology/kei047.

2. Khan, M.A. Polymorphism of HLA-B27: 105 subtypes currently known. Curr Rheumatol Rep 2013, 15, 362, doi:10.1007/s11926-013-0362-y.

3. Stolwijk, C.; Boonen, A.; van Tubergen, A.; Reveille, J.D. Epidemiology of spondyloarthritis. Rheum Dis Clin North Am 2012, 38, 441-476, doi:10.1016/j.rdc.2012.09.003.

4. Bakland, G.; Nossent, H.C. Epidemiology of Spondyloarthritis: A Review. Current Rheumatology Reports 2013, 15, 351, doi:10.1007/s11926-013-0351-1.

5. Braun, J.; Sieper, J. Ankylosing spondylitis. The Lancet 2007, 369, 1379-1390, doi:10.1016/S0140-6736(07)60635-7.

6. Gotch, F.; Rothbard, J.; Howland, K.; Townsend, A.; McMichael, A. Cytotoxic $\mathrm{T}$ lymphocytes recognize a fragment of influenza virus matrix protein in association with HLA-A2. Nature 1987, 326, 881-882, doi:10.1038/326881a0. 
7. Townsend, A.R.M.; Rothbard, J.; Gotch, F.M.; Bahadur, G.; Wraith, D.; McMichael, A.J. The epitopes of influenza nucleoprotein recognized by cytotoxic T lymphocytes can be defined with short synthetic peptides. Cell 1986, 44, 959-968, doi:https://doi.org/10.1016/0092-8674(86)90019-X.

8. Colbert, R.A.; Tran, T.M.; Layh-Schmitt, G. HLA-B27 misfolding and ankylosing spondylitis. Mol Immunol 2014, 57, 44-51, doi:10.1016/j.molimm.2013.07.013.

9. Trowsdale, J.; Knight, J.C. Major histocompatibility complex genomics and human disease. Annu Rev Genomics Hum Genet 2013, 14, 301-323, doi:10.1146/annurev-genom-091212-153455.

10. Bjorkman, P.; Parham, P. Structure, Function, and Diversity of Class I Major Histocompatibility Complex Molecules. Annual review of biochemistry 1990, 59, 253-288, doi:10.1146/annurev.bi.59.070190.001345.

11. Crux, N.; Elahi, S. Human Leukocyte Antigen (HLA) and Immune Regulation: How Do Classical and Non-Classical HLA Alleles Modulate Immune Response to Human Immunodeficiency Virus and Hepatitis C Virus Infections? Frontiers in Immunology 2017, 8, doi:10.3389/fimmu.2017.00832.

12. McMichael, A.; Bowness, P. HLA-B27: natural function and pathogenic role in spondyloarthritis. Arthritis Res 2002, 4 Suppl 3, S153-158, doi:10.1186/ar571.

13. Reveille, J.D. 57 - Spondyloarthritis. In Clinical Immunology (Fifth Edition), Rich, R.R., Fleisher, T.A., Shearer, W.T., Schroeder, H.W., Frew, A.J., Weyand, C.M., Eds. Content Repository Only!: London, 2019; https://doi.org/10.1016/B978-07020-6896-6.00057-0pp. 769-787.

14. Hill Gaston, J.S.; Lillicrap, M.S. Arthritis associated with enteric infection. Best Pract Res Clin Rheumatol 2003, 17, 219-239, doi:10.1016/s1521-6942(02)00104-3.

15. Sieper, J.; Braun, J.; Kingsley, G.H. Report on the Fourth International Workshop on Reactive Arthritis. Arthritis Rheum 2000, 43, 720-734, doi:10.1002/1529-0131(200004)43:4<720::AID-ANR2>3.0.CO;2-8. 
16. Granfors, K.; Jalkanen, S.; von Essen, R.; Lahesmaa-Rantala, R.; Isomäki, O.; Pekkola-Heino, K.; Merilahti-Palo, R.; Saario, R.; Isomäki, H.; Toivanen, A. Yersinia Antigens in Synovial-Fluid Cells from Patients with Reactive Arthritis. New England Journal of Medicine 1989, 320, 216-221, doi:10.1056/NEJM198901263200404.

17. Allen, R.L.; Bowness, P.; McMichael, A. The role of HLA-B27 in spondyloarthritis. Immunogenetics 1999, 50, 220-227, doi:10.1007/s002510050596.

18. Zhu, W.; He, X.; Cheng, K.; Zhang, L.; Chen, D.; Wang, X.; Qiu, G.; Cao, X.; Weng, X. Ankylosing spondylitis: etiology, pathogenesis, and treatments. Bone Res 2019, 7, 22, doi:10.1038/s41413-019-0057-8.

19. Oldstone, M.B.A. Molecular Mimicry as a Mechanism for the Cause and as a Probe Uncovering Etiologic Agent(S) of Autoimmune-Disease. Current Topics in Microbiology and Immunology 1989, 145, 127-135.

20. Grandon, B.; Rincheval-Arnold, A.; Jah, N.; Corsi, J.M.; Araujo, L.M.; Glatigny, S.; Prevost, E.; Roche, D.; Chiocchia, G.; Guenal, I., et al. HLA-B27 alters BMP/TGFbeta signalling in Drosophila, revealing putative pathogenic mechanism for spondyloarthritis. Ann Rheum Dis 2019, 78, 1653-1662, doi:10.1136/annrheumdis-2019-215832.

21. Pedersen, S.J.; Maksymowych, W.P. The Pathogenesis of Ankylosing Spondylitis: an Update. Curr Rheumatol Rep 2019, 21, 58, doi:10.1007/s11926019-0856-3.

22. Garcia-Montoya, L.; Gul, H.; Emery, P. Recent advances in ankylosing spondylitis: understanding the disease and management. F1000Res 2018, 7, doi:10.12688/f1000research.14956.1.

23. Martin-Esteban, A.; Sanz-Bravo, A.; Guasp, P.; Barnea, E.; Admon, A.; de Castro, J.A.L. Separate effects of the ankylosing spondylitis associated ERAP1 and ERAP2 aminopeptidases determine the influence of their combined phenotype on the HLA-B*27 peptidome. Journal of Autoimmunity 2017, 79, 28 38, doi:10.1016/j.jaut.2016.12.008. 
24. Babaie, F.; Mohammadi, H.; Hemmatzadeh, M.; Ebrazeh, M.; Torkamandi, S.; Yousefi, M.; Hajaliloo, M.; Rezaiemanesh, A.; Salimi, S.; Salimi, R., et al. Evaluation of ERAP1 gene single nucleotide polymorphisms in immunomodulation of pro-inflammatory and anti-inflammatory cytokines profile in ankylosing spondylitis. Immunol Lett 2020, 217, 31-38, doi:10.1016/j.imlet.2019.10.016.

25. Stoll, M.L. Interactions of the innate and adaptive arms of the immune system in the pathogenesis of spondyloarthritis. Clin Exp Rheumatol 2011, 29, 322-330.

26. Cusick, M.F.; Libbey, J.E.; Fujinami, R.S. Molecular Mimicry as a Mechanism of Autoimmune Disease. Clinical Reviews in Allergy E Immunology 2012, 42, $102-$ 111, doi:10.1007/s12016-011-8294-7.

27. Kuon, W.; Holzhutter, H.G.; Appel, H.; Grolms, M.; Kollnberger, S.; Traeder, A.; Henklein, P.; Weiss, E.; Thiel, A.; Lauster, R., et al. Identification of HLAB27-restricted peptides from the Chlamydia trachomatis proteome with possible relevance to HLA-B27-associated diseases. J Immunol 2001, 167, 47384746, doi:10.4049/jimmunol.167.8.4738.

28. Ramos, M.; Lopez de Castro, J.A. HLA-B27 and the pathogenesis of spondyloarthritis. Tissue Antigens 2002, 60, 191-205, doi:10.1034/j.13990039.2002.600301.x.

29. Bowness, P. Hla-B27. Annual Review of Immunology Vol 33 2015, 33, 29-48, doi:10.1146/annurev-immunol-032414-112110.

30. Appel, H.; Kuon, W.; Kuhne, M.; Wu, P.H.; Kuhlmann, S.; Kollnberger, S.; Thiel, A.; Bowness, P.; Sieper, J. Use of HLA-B27 tetramers to identify low-frequency antigen-specific T cells in Chlamydia-triggered reactive arthritis. Arthritis Research $\mathcal{E}$ Therapy 2004, 6, R521-R534, doi:10.1186/ar1221.

31. Hermann, E.; Yu, D.T.Y.; Zumbuschenfelde, K.H.M.; Fleischer, B. Hla-B27Restricted Cd8 T-Cells Derived from Synovial-Fluids of Patients with Reactive Arthritis and Ankylosing-Spondylitis. Lancet 1993, 342, 646-650, doi:Doi 10.1016/0140-6736(93)91760-J. 
32. Ugrinovic, S.; Mertz, A.; Wu, P.; Braun, J.; Sieper, J. A single nonamer from the Yersinia $60-\mathrm{kDa}$ heat shock protein is the target of HLA-B27-restricted CTL response in Yersinia-induced reactive arthritis. J Immunol 1997, 159, 5715-5723.

33. Scofield, R.H.; Kurien, B.; Gross, T.; Warren, W.L.; Harley, J.B. HLA-B27 binding of peptide from its own sequence and similar peptides from bacteria: implications for spondyloarthropathies. Lancet 1995, 345, 1542-1544, doi:10.1016/s0140-6736(95)91089-1.

34. Ewing, C.; Ebringer, R.; Tribbick, G.; Geysen, H.M. Antibody activity in ankylosing spondylitis sera to two sites on HLA B27.1 at the MHC groove region (within sequence 65-85), and to a Klebsiella pneumoniae nitrogenase reductase peptide (within sequence 181-199). J Exp Med 1990, 171, 1635-1647, doi:10.1084/jem.171.5.1635.

35. Ben Dror, L.; Barnea, E.; Beer, I.; Mann, M.; Admon, A. The HLA-B*2705 peptidome. Arthritis Rheum 2010, 62, 420-429, doi:10.1002/art.27257.

36. Fiorillo, M.T.; Maragno, M.; Butler, R.; Dupuis, M.L.; Sorrentino, R. CD8(+) Tcell autoreactivity to an HLA-B27-restricted self-epitope correlates with ankylosing spondylitis. J Clin Invest 2000, 106, 47-53, doi:10.1172/JCI9295.

37. Syrbe, U.; Sieper, J. Chapter 36 - Spondyloarthritides. In The Autoimmune Diseases (Sixth Edition), Rose, N.R., Mackay, I.R., Eds. Academic Press: 2020; https://doi.org/10.1016/B978-0-12-812102-3.00036-1pp. 691-701.

38. Schittenhelm, R.B.; Sian, T.C.; Wilmann, P.G.; Dudek, N.L.; Purcell, A.W. Revisiting the arthritogenic peptide theory: quantitative not qualitative changes in the peptide repertoire of HLA-B27 allotypes. Arthritis Rheumatol 2015, 67, 702-713, doi:10.1002/art.38963.

39. Babaie, F.; Hasankhani, M.; Mohammadi, H.; Safarzadeh, E.; Rezaiemanesh, A.; Salimi, R.; Baradaran, B.; Babaloo, Z. The role of gut microbiota and IL-23/IL17 pathway in ankylosing spondylitis immunopathogenesis: New insights and updates. Immunol Lett 2018, 196, 52-62, doi:10.1016/j.imlet.2018.01.014. 
40. Mear, J.P.; Schreiber, K.L.; Munz, C.; Zhu, X.M.; Stevanovic, S.; Rammensee, H.G.; Rowland-Jones, S.L.; Colbert, R.A. Misfolding of HLA-B27 as a result of its B pocket suggests a novel mechanism for its role in susceptibility to spondyloarthropathies. Journal of Immunology 1999, 163, 6665-6670.

41. Kenna, T.J.; Robinson, P.C.; Haroon, N. Endoplasmic reticulum aminopeptidases in the pathogenesis of ankylosing spondylitis. Rheumatology 2015, 54, 1549-1556, doi:10.1093/rheumatology/kev218.

42. Ambarus, C.A.; Yeremenko, N.; Baeten, D.L. Altered cytokine expression by macrophages from HLA-B27-positive spondyloarthritis patients without evidence of endoplasmic reticulum stress. Rheumatol Adv Pract 2018, 2, rky014, doi:10.1093/rap/rky014.

43. Smith, J.A. Regulation of Cytokine Production by the Unfolded Protein Response; Implications for Infection and Autoimmunity. Front Immunol 2018, 9, 422, doi:10.3389/fimmu.2018.00422.

44. Busch, R.; Kollnberger, S.; Mellins, E.D. HLA associations in inflammatory arthritis: emerging mechanisms and clinical implications. Nat Rev Rheumatol 2019, 15, 364-381, doi:10.1038/s41584-019-0219-5.

45. Navid, F.; Layh-Schmitt, G.; Sikora, K.A.; Cougnoux, A.; Colbert, R.A. The Role of Autophagy in the Degradation of Misfolded HLA-B27 Heavy Chains. Arthritis Rheumatol 2018, 70, 746-755, doi:10.1002/art.40414.

46. Khare, S.D.; Luthra, H.S.; David, C.S. Spontaneous inflammatory arthritis in HLA-B27 transgenic mice lacking beta 2-microglobulin: a model of human spondyloarthropathies. J Exp Med 1995, 182, 1153-1158, doi:10.1084/jem.182.4.1153.

47. Ramos, M.; de Castro, J.A.L. HLA-B27 and the pathogenesis of spondyloarthritis. Tissue Antigens 2002, 60, 191-205, doi:DOI 10.1034/j.13990039.2002.600301.x.

48. Ebringer, A. The Cross-Tolerance Hypothesis, Hla-B27 and AnkylosingSpondylitis. Brit J Rheumatol 1983, 22, 53-66. 
49. Turner, M.J.; Delay, M.L.; Bai, S.; Klenk, E.; Colbert, R.A. HLA-B27 upregulation causes accumulation of misfolded heavy chains and correlates with the magnitude of the unfolded protein response in transgenic rats: Implications for the pathogenesis of spondylarthritis-like disease. Arthritis Rheum 2007, 56, 215-223, doi:10.1002/art.22295.

50. Delay, M.L.; Turner, M.J.; Klenk, E.I.; Smith, J.A.; Sowders, D.P.; Colbert, R.A. HLA-B27 Misfolding and the Unfolded Protein Response Augment Interleukin-23 Production and Are Associated With Th17 Activation in Transgenic Rats. Arthritis Rheum-Us 2009, 60, 2633-2643, doi:10.1002/art.24763.

51. Tran, T.M.; Dorris, M.L.; Satumtira, N.; Richardson, J.A.; Hammer, R.E.; Shang, J.; Taurog, J.D. Additional human beta(2)-microglobulin curbs HLA-B27 misfolding and promotes arthritis and spondylitis without colitis in male HLAB27-transgenic rats. Arthritis Rheum-Us 2006, 54, 1317-1327, doi:10.1002/art.21740.

52. Campbell, E.C.; Fettke, F.; Bhat, S.; Morley, K.D.; Powis, S.J. Expression of MHC class I dimers and ERAP1 in an ankylosing spondylitis patient cohort. Immunology 2011, 133, 379-385, doi:10.1111/j.1365-2567.2011.03453.x.

53. Ciccia, F.; Accardo-Palumbo, A.; Rizzo, A.; Guggino, G.; Raimondo, S.; Giardina, A.; Cannizzaro, A.; Colbert, R.A.; Alessandro, R.; Triolo, G. Evidence that autophagy, but not the unfolded protein response, regulates the expression of IL-23 in the gut of patients with ankylosing spondylitis and subclinical gut inflammation. Annals of the Rheumatic Diseases 2014, 73, 1566-1574, doi:10.1136/annrheumdis-2012-202925.

54. Rezaiemanesh, A.; Mahmoudi, M.; Amirzargar, A.A.; Vojdanian, M.; Jamshidi, A.R.; Nicknam, M.H. Ankylosing spondylitis M-CSF-derived macrophages are undergoing unfolded protein response (UPR) and express higher levels of interleukin-23. Mod Rheumatol 2017, 27, 862-867, doi:10.1080/14397595.2016.1259716. 
55. Taurog, J.D.; Dorris Ml Fau - Satumtira, N.; Satumtira N Fau - Tran, T.M.; Tran Tm Fau - Sharma, R.; Sharma R Fau - Dressel, R.; Dressel R Fau - van den Brandt, J.; van den Brandt J Fau - Reichardt, H.M.; Reichardt, H.M. Spondylarthritis in HLA-B27/human beta2-microglobulin-transgenic rats is not prevented by lack of CD8.

56. Boyle, L.H.; Hill Gaston, J.S. Breaking the rules: the unconventional recognition of HLA-B27 by CD4+ T lymphocytes as an insight into the pathogenesis of the spondyloarthropathies. Rheumatology 2003, 42, 404-412, doi:10.1093/rheumatology/keg097.

57. Kollnberger, S.; Bird, L.; Sun, M.-Y.; Retiere, C.; Braud, V.M.; McMichael, A.; Bowness, P. Cell-surface expression and immune receptor recognition of HLAB27 homodimers. Arthritis \& Rheumatism 2002, 46, 2972-2982, doi:10.1002/art.10605.

58. Antoniou, A.N.; Ford, S.; Taurog, J.D.; Butcher, G.W.; Powis, S.J. Formation of HLA-B27 homodimers and their relationship to assembly kinetics. Journal of Biological Chemistry 2004, 279, 8895-8902, doi:10.1074/jbc.M311757200.

59. Brown, M.A. Solving the pathogenesis of ankylosing spondylitis. Clin Immunol 2018, 186, 46-50, doi:10.1016/j.clim.2017.07.011.

60. Bowness, P.; Ridley, A.; Shaw, J.; Chan, A.T.; Wong-Baeza, I.; Fleming, M.; Cummings, F.; McMichael, A.; Kollnberger, S. Th17 cells expressing KIR3DL2+ and responsive to HLA-B27 homodimers are increased in ankylosing spondylitis. J Immunol 2011, 186, 2672-2680, doi:10.4049/jimmunol.1002653.

61. Chen, L.; Ridley, A.; A, H.; Al-Mossawi, H.; Bunting, H.; Georgiadis, D.; Chan, A.; Kollnberger, S.; Bowness, P. Silencing or inhibition of endoplasmic reticulum aminopeptidase 1 (ERAP1) suppresses free heavy chain expression and Th17 responses in ankylosing spondylitis. Annals of the Rheumatic Diseases 2015, 75, doi:10.1136/annrheumdis-2014-206996.

62. Babaie, F.; Ebrazeh, M.; Hemmatzadeh, M.; Sadat Mohammadi, F.; Gowhari Shabgah, A.; Hajaliloo, M.; Ebrahimi, A.A.; Shirafkan, N.; Azizi, G.; 
Mohammadi, H., et al. Association analysis of ERAP1 gene single nucleotide polymorphism in susceptibility to ankylosing spondylitis in Iranian population. Immunol Lett 2018, 201, 52-58, doi:10.1016/j.imlet.2018.11.002.

63. Giles, J.; Shaw J Fau - Piper, C.; Piper C Fau - Wong-Baeza, I.; Wong-Baeza I Fau - McHugh, K.; McHugh K Fau - Ridley, A.; Ridley A Fau - Li, D.; Li D Fau - Lenart, I.; Lenart I Fau - Antoniou, A.N.; Antoniou An Fau - DiGleria, K.; DiGleria K Fau - Kuroki, K., et al. HLA-B27 homodimers and free H chains are stronger ligands for leukocyte Ig-like receptor B2 than classical HLA class I. D - NLM: EMS52285 EDAT- 2012/05/18 06:00 MHDA- 2012/08/15 06:00 CRDT2012/05/18 06:00 PHST- 2012/05/18 06:00 [entrez] PHST- 2012/05/18 06:00 [pubmed] PHST- 2012/08/15 06:00 [medline] AID - jimmunol.1102711 [pii] AID - 10.4049/jimmunol.1102711 [doi] PST - ppublish, doi:D - NLM: EMS52285 EDAT- 2012/05/18 06:00 MHDA- 2012/08/15 06:00 CRDT- 2012/05/18 06:00 PHST- 2012/05/18 06:00 [entrez] PHST- 2012/05/18 06:00 [pubmed] PHST2012/08/15 06:00 [medline] AID - jimmunol.1102711 [pii] AID 10.4049/jimmunol.1102711 [doi] PST - ppublish.

64. Chan, A.T.; Kollnberger Sd Fau - Wedderburn, L.R.; Wedderburn Lr Fau Bowness, P.; Bowness, P. Expansion and enhanced survival of natural killer cells expressing the killer immunoglobulin-like receptor KIR3DL2 in spondylarthritis.

65. Cauli, A.; Shaw, J.; Giles, J.; Hatano, H.; Rysnik, O.; Payeli, S.; McHugh, K.; Dessole, G.; Porru, G.; Desogus, E., et al. The arthritis-associated HLA-B²7:05 allele forms more cell surface B27 dimer and free heavy chain ligands for KIR3DL2 than HLA-B*27:09. Rheumatology (Oxford, England) 2013, 52, 1952 1962, doi:10.1093/rheumatology/ket219.

66. Lim Kam Sian, T.C.C.; Indumathy, S.; Halim, H.; Greule, A.; Cryle, M.J.; Bowness, P.; Rossjohn, J.; Gras, S.; Purcell, A.W.; Schittenhelm, R.B. Allelic association with ankylosing spondylitis fails to correlate with human leukocyte 
antigen B27 homodimer formation. J Biol Chem 2019, 294, 20185-20195, doi:10.1074/jbc.RA119.010257.

67. Evans, D.M.; Spencer, C.C.A.; Pointon, J.J.; Su, Z.; Harvey, D.; Kochan, G.; Opperman, U.; Dilthey, A.; Pirinen, M.; Stone, M.A., et al. Interaction between ERAP1 and HLA-B27 in ankylosing spondylitis implicates peptide handling in the mechanism for HLA-B27 in disease susceptibility. Nature Genetics 2011, 43, 761-U767, doi:10.1038/ng.873.

68. Mahmoudi, M.; Jamshidi, A.R.; Amirzargar, A.A.; Farhadi, E.; Nourijelyani, K.; Fallahi, S.; Oraei, M.; Noori, S.; Nicknam, M.H. Association between Endoplasmic Reticulum Aminopeptidase-1 (ERAP-1) and Susceptibility to Ankylosing Spondylitis in Iran. Iranian Journal of Allergy Asthma and Immunology 2012, 11, 294-300.

69. Dashti, N.; Mahmoudi, M.; Aslani, S.; Jamshidi, A. HLA-B²7 subtypes and their implications in the pathogenesis of ankylosing spondylitis. Gene 2018, 670, 15-21, doi:https://doi.org/10.1016/j.gene.2018.05.092.

70. Davidson, S.I.; Wu X Fau - Liu, Y.; Liu Y Fau - Wei, M.; Wei M Fau - Danoy, P.A.; Danoy Pa Fau - Thomas, G.; Thomas G Fau - Cai, Q.; Cai Q Fau - Sun, L.; Sun L Fau - Duncan, E.; Duncan E Fau - Wang, N.; Wang N Fau - Yu, Q., et al. Association of ERAP1, but not IL23R, with ankylosing spondylitis in a Han Chinese population.

71. Burton, P.; Clayton, D.; Cardon, L.; Craddock, N.; Duncanson, A.; Kwiatkowski, D.; McCarthy, M.; Ouwehand, W.; Samani, N.; Todd, J., et al. Association scan of 14,500 nonsynonymous SNPs in four diseases identifies autoimmunity variants. Nature genetics 2007, 39, 1329-1337, doi:10.1038/ng.2007.17.

72. Brown, M.A. Breakthroughs in genetic studies of ankylosing spondylitis. Rheumatology 2007, 47, 132-137, doi:10.1093/rheumatology/kem269.

73. Saric, T.; Chang, S.C.; Hattori, A.; York, I.A.; Markant, S.; Rock, K.L.; Tsujimoto, M.; Goldberg, A.L. An IFN-gamma-induced aminopeptidase in the ER, ERAP1, 
trims precursors to MHC class I-presented peptides. Nat Immunol 2002, 3, 11691176, doi:10.1038/ni859.

74. Saveanu, L.; van Endert, P. The role of insulin-regulated aminopeptidase in MHC class I antigen presentation. Front Immunol 2012, 3, 57, doi:10.3389/fimmu.2012.00057.

75. McHugh, K.; Bowness, P. The link between HLA-B27 and SpA--new ideas on an old problem. Rheumatology (Oxford, England) 2012, 51, 1529-1539, doi:10.1093/rheumatology/kes061.

76. Cui, X.L.; Hawari, F.; Alsaaty, S.; Lawrence, M.; Combs, C.A.; Geng, W.D.; Rouhani, F.N.; Miskinis, D.; Levine, S.J. Identification of ARTS-1 as a novel TNFR1-binding protein that promotes TNFR1 ectodomain shedding. Journal of Clinical Investigation 2002, 110, 515-526, doi:10.1172/Jci200213847.

77. Reveille, J.D. Genetics of spondyloarthritis - beyond the MHC. Nature Reviews Rheumatology 2012, 8, 296-304, doi:10.1038/nrrheum.2012.41.

78. Keidel, S.; Chen, L.; Pointon, J.; Wordsworth, P. ERAP1 and ankylosing spondylitis. Curr Opin Immunol 2013, 25, 97-102, doi:10.1016/j.coi.2012.11.002.

79. Kirino, Y.; Bertsias, G.; Ishigatsubo, Y.; Mizuki, N.; Tugal-Tutkun, I.; Seyahi, E.; Ozyazgan, Y.; Sacli, F.S.; Erer, B.; Inoko, H., et al. Genome-wide association analysis identifies new susceptibility loci for Behcet's disease and epistasis between HLA-B*51 and ERAP1. Nat Genet 2013, 45, 202-207, doi:10.1038/ng.2520.

80. Genetic Analysis of Psoriasis, C.; the Wellcome Trust Case Control, C.; Strange, A.; Capon, F.; Spencer, C.C.A.; Knight, J.; Weale, M.E.; Allen, M.H.; Barton, A.; Band, G., et al. A genome-wide association study identifies new psoriasis susceptibility loci and an interaction between HLA-C and ERAP1. Nature genetics 2010, 42, 985-990, doi:10.1038/ng.694.

81. Kochan, G.; Krojer, T.; Harvey, D.; Fischer, R.; Chen, L.; Vollmar, M.; von Delft, F.; Kavanagh, K.L.; Brown, M.A.; Bowness, P., et al. Crystal structures of the endoplasmic reticulum aminopeptidase-1 (ERAP1) reveal the molecular basis 
for N-terminal peptide trimming. Proc Natl Acad Sci U S A 2011, 108, 7745-7750, doi:10.1073/pnas.1101262108.

82. Li, L.; Batliwala, M.; Bouvier, M. ERAP1 enzyme-mediated trimming and structural analyses of MHC I--bound precursor peptides yield novel insights into antigen processing and presentation. Journal of Biological Chemistry 2019, 294, jbc.RA119.010102, doi:10.1074/jbc.RA119.010102.

83. Seregin, S.S.; Rastall, D.P.; Evnouchidou, I.; Aylsworth, C.F.; Quiroga, D.; Kamal, R.P.; Godbehere-Roosa, S.; Blum, C.F.; York, I.A.; Stratikos, E., et al. Endoplasmic reticulum aminopeptidase-1 alleles associated with increased risk of ankylosing spondylitis reduce HLA-B27 mediated presentation of multiple antigens. Autoimmunity 2013, 46, 497-508, doi:10.3109/08916934.2013.819855.

84. Tang, Y.; Yang, P.; Wang, F.; Xu, H.; Zong, S.Y. Association of polymorphisms in ERAP1 and risk of ankylosing spondylitis in a Chinese population. Gene 2018, 646, 8-11, doi:10.1016/j.gene.2017.12.050.

85. Wisniewski, A.; Kasprzyk, S.; Majorczyk, E.; Nowak, I.; Wilczynska, K.; Chlebicki, A.; Zon-Giebel, A.; Kusnierczyk, P. ERAP1-ERAP2 haplotypes are associated with ankylosing spondylitis in Polish patients. Hum Immunol 2019, 80, 339-343, doi:10.1016/j.humimm.2019.02.004.

86. Paladini, F.; Fiorillo, M.T.; Tedeschi, V.; D'Otolo, V.; Piga, M.; Cauli, A.; Mathieu, A.; Sorrentino, R. The rs75862629 minor allele in the endoplasmic reticulum aminopeptidases intergenic region affects human leucocyte antigen B27 expression and protects from ankylosing spondylitis in Sardinia. Rheumatology (Oxford) 2019, 58, 2315-2324, doi:10.1093/rheumatology/kez212.

87. Hammer, G.E.; Gonzalez, F.; Champsaur, M.; Cado, D.; Shastri, N. The aminopeptidase ERAAP shapes the peptide repertoire displayed by major histocompatibility complex class I molecules. Nat Immunol 2006, 7, 103-112, doi:10.1038/ni1286.

88. Haroon, N.; Tsui, F.; Chiu, B.; Tsui, H.; Inman, R. Serum Cytokine Receptors in Ankylosing Spondylitis: Relationship to Inflammatory Markers and 
Endoplasmic Reticulum Aminopeptidase Polymorphisms. The Journal of rheumatology 2010, 37, 1907-1910, doi:10.3899/jrheum.100019.

89. Chen, B.; Li, D.; Xu, W. Association of ankylosing spondylitis with HLA-B27 and ERAP1: pathogenic role of antigenic peptide. Med Hypotheses 2013, 80, 3638, doi:10.1016/j.mehy.2012.10.003.

90. Gill, T.; Asquith, M.; Brooks, S.R.; Rosenbaum, J.T.; Colbert, R.A. Effects of HLA-B27 on Gut Microbiota in Experimental Spondyloarthritis Implicate an Ecological Model of Dysbiosis. Arthritis Rheumatol 2018, 70, 555-565, doi:10.1002/art.40405.

91. Antoniou, A.N.; Lenart, I.; Kriston-Vizi, J.; Iwawaki, T.; Turmaine, M.; McHugh, K.; Ali, S.; Blake, N.; Bowness, P.; Bajaj-Elliott, M., et al. Salmonella exploits HLA-B27 and host unfolded protein responses to promote intracellular replication. Ann Rheum Dis 2019, 78, 74-82, doi:10.1136/annrheumdis-2018213532.

92. Ansalone, C.; Utriainen, L.; Milling, S.; Goodyear, C.S. Role of Gut Inflammation in Altering the Monocyte Compartment and Its Osteoclastogenic Potential in HLA-B27-Transgenic Rats. Arthritis Rheumatol 2017, 69, 1807-1815, doi:10.1002/art.40154.

93. Ombrello, M.J.; Remmers, E.F.; Tachmazidou, I.; Grom, A.; Foell, D.; Haas, J.P.; Martini, A.; Gattorno, M.; Ozen, S.; Prahalad, S., et al. HLA-DRB1*11 and variants of the MHC class II locus are strong risk factors for systemic juvenile idiopathic arthritis. Proc Natl Acad Sci U S A 2015, 112, 15970-15975, doi:10.1073/pnas.1520779112.

94. Lin, P.; Bach, M.; Asquith, M.; Lee, A.Y.; Akileswaran, L.; Stauffer, P.; Davin, S.; Pan, Y.; Cambronne, E.D.; Dorris, M., et al. HLA-B27 and human beta2microglobulin affect the gut microbiota of transgenic rats. PLoS One 2014, 9, e105684, doi:10.1371/journal.pone.0105684. 
95. Rosenbaum, J.T.; Davey, M.P. Time for a gut check: evidence for the hypothesis that HLA-B27 predisposes to ankylosing spondylitis by altering the microbiome. Arthritis Rheum 2011, 63, 3195-3198, doi:10.1002/art.30558.

96. Asquith, M.J.; Stauffer, P.; Davin, S.; Mitchell, C.; Lin, P.; Rosenbaum, J.T. Perturbed Mucosal Immunity and Dysbiosis Accompany Clinical Disease in a Rat Model of Spondyloarthritis. Arthritis Rheumatol 2016, 68, 2151-2162, doi:10.1002/art.39681.

97. Sartor, R.B.; Wu, G.D. Roles for Intestinal Bacteria, Viruses, and Fungi in Pathogenesis of Inflammatory Bowel Diseases and Therapeutic Approaches. Gastroenterology 2017, 152, 327-339 e324, doi:10.1053/j.gastro.2016.10.012.

98. Rizzo, A.; Ferrante, A.; Guggino, G.; Ciccia, F. Gut inflammation in spondyloarthritis. Best Pract Res Clin Rheumatol 2017, 31, 863-876, doi:10.1016/j.berh.2018.08.012.

99. Reid, G.; Younes, J.A.; Van der Mei, H.C.; Gloor, G.B.; Knight, R.; Busscher, H.J. Microbiota restoration: natural and supplemented recovery of human microbial communities. Nat Rev Microbiol 2011, 9, 27-38, doi:10.1038/nrmicro2473.

100. Mielants, H.; Veys, E.M.; De Vos, M.; Cuvelier, C. Increased intestinal permeability in ankylosing spondylitis. Gut 1992, 33, 1150, doi:10.1136/gut.33.8.1150.

101. Costello, M.E.; Ciccia, F.; Willner, D.; Warrington, N.; Robinson, P.C.; Gardiner, B.; Marshall, M.; Kenna, T.J.; Triolo, G.; Brown, M.A. Brief Report: Intestinal Dysbiosis in Ankylosing Spondylitis. Arthritis Rheumatol 2015, 67, 686-691, doi:10.1002/art.38967.

102. Zhang, L.; Hu, Y.; Xu, Y.; Li, P.; Ma, H.; Li, X.; Li, M. The correlation between intestinal dysbiosis and the development of ankylosing spondylitis. Microbial Pathogenesis 2019, 132, 188-192, doi:https://doi.org/10.1016/j.micpath.2019.04.038. 
103. Breban, M.; Tap, J.; Leboime, A.; Said-Nahal, R.; Langella, P.; Chiocchia, G.; Furet, J.P.; Sokol, H. Faecal microbiota study reveals specific dysbiosis in spondyloarthritis. Ann Rheum Dis 2017, 76, 1614-1622, doi:10.1136/annrheumdis-2016-211064.

104. Wen, C.; Zheng, Z.; Shao, T.; Liu, L.; Xie, Z.; Le Chatelier, E.; He, Z.; Zhong, W.; Fan, Y.; Zhang, L., et al. Quantitative metagenomics reveals unique gut microbiome biomarkers in ankylosing spondylitis. Genome Biol 2017, 18, 142, doi:10.1186/s13059-017-1271-6.

105. Yin, J.; Sternes, P.R.; Wang, M.; Song, J.; Morrison, M.; Li, T.; Zhou, L.; Wu, X.; He, F.; Zhu, J., et al. Shotgun metagenomics reveals an enrichment of potentially cross-reactive bacterial epitopes in ankylosing spondylitis patients, as well as the effects of TNFi therapy upon microbiome composition. Annals of the Rheumatic Diseases 2020, 79, 132, doi:10.1136/annrheumdis-2019-215763. 